\title{
Synthetic oligonucleotides: AFM characterisation and electroanalytical studies
}

\author{
A.M. Oliveira Brett ${ }^{\mathrm{a}, *}$, A.-M. Chiorcea Paquim ${ }^{\mathrm{a}}$, V. Diculescu ${ }^{\mathrm{a}}$, T.S. Oretskaya ${ }^{\mathrm{b}}$ \\ ${ }^{a}$ Departamento de Quimica, Faculdade de Ciências e Tecnologia, Universidade de Coimbra, 3004-535 Coimbra, Portugal \\ ${ }^{\mathrm{b}}$ Chemistry Department, Moscow State University, Vorobjovy Gory, 119899, Moscow, Russia
}

Received 15 January 2004; received in revised form 10 June 2004; accepted 15 June 2004

Available online 21 July 2005

\begin{abstract}
One of the most important steps in designing more sensitive and stable DNA based biosensors is the immobilisation procedure of the nucleic acid probes on the transducer surface, while maintaining their conformational flexibility. MAC Mode AFM images in air demonstrated that the oligonucleotide sequences adsorb spontaneously on the electrode surface, showing the existence of pores in the adsorbed layer that reveal big parts of the electrode surface, which enables non-specific adsorption of other molecules on the uncovered areas. The electrostatic immobilisation onto a glassy carbon electrode followed by hybridisation with a complementary sequence and control with a non-complementary sequence was studied using differential pulse voltammetry and electrochemical impedance spectroscopy. Changes in the oxidation currents of guanosine and adenosine were observed after hybridisation events as well as after control experiments. Modification of the double layer capacitance that took place after hybridisation or control experiments showed that non-specific adsorption of complementary or non-complementary sequences occur allowing the formation of a mixed multilayer.
\end{abstract}

(C) 2005 Elsevier B.V. All rights reserved.

Keywords: DNA; DNA biosensor; Hybridisation; Adsorption; Non-specific adsorption; AFM

\section{Introduction}

In recent years an increasing interest in developing small and portable DNA-biosensor devices using molecular recognition of hybridisation events was observed. The DNA sensor combines the specificity of the DNA biological recognition mechanism with a physical transduction technique, which can be based either on electrochemical [1-12], mechanical [13-15], thermal $[16,17]$ or optical sensing principles [18-20].

Nucleic acid based biosensors normally employ immobilized DNA sequences on the sensor surface as the recognition element and sequence specific hybridisation can be monitored and analysed. Short, synthetic oligonucleotides are ideal chemical recognition elements, because the hybridisation is highly sequence-selective.

\footnotetext{
* Corresponding author. Tel./fax: +351 239835295.

E-mail address: brett@ci.uc.pt (A.M. Oliveira Brett).
}

The hybridisation strategy at a DNA biosensor consists essentially of three steps: the immobilisation of the oligonucleotide probe at the transducer surface, the hybridisation with the complementary strand (target) present in the solution and the transduction. The first step of immobilisation of the oligonucleotide probe enables the modification of the chemical and structural properties of the sensor, playing the most important role in the performance of the DNA biosensor.

Careful attention has been paid to different factors such as substrate material, DNA nature, immobilisation density, and a variety of experimental conditions, temperature, $\mathrm{pH}$ or ionic strength, as well as sample processing in order to increase the selectivity, sensitivity or final speed of the hybridisation assay [21].

Magnetic AC mode Atomic Force Microscopy (MAC Mode AFM) is a gentle technique that permits the investigation of DNA molecules, which are loosely attached to conducting electrode surfaces $[22,23]$. MAC Mode AFM 
perturbation of $5 \mathrm{mV}$ was applied over the frequency range $100 \mathrm{kHz}-0.1 \mathrm{~Hz}$ with five frequency values per decade and each spectrum was recorded at $+0.5 \mathrm{~V}$ vs. 7 $\mathrm{Ag} / \mathrm{AgCl}$.

All the plots were treated and presented in Microcal Origin Version 6.0.

\subsection{Atomic force microscopy and electrochemical experimental procedures}

For AFM surface characterization, $500 \mu \mathrm{L}$ of the desired oligonucleotide solution were placed in the electrochemical cell holding the HOPG working electrode on the base. A positive potential of $+0.65 \mathrm{~V}$, vs. AgQRE, was applied to the electrode for 180 or $300 \mathrm{~s}$. The HOPG with adsorbed oligonucleotide molecules was rinsed with a jet of Millipore Milli-Q water and dried in a sterile atmosphere.

The electrochemical hybridisation experiments were carried out at room temperature following a procedure that consists of four steps: probe immobilisation, hybridisation, transduction and control.

\subsubsection{Probe immobilisation}

A freshly polished GCE was cycled in differential pulse voltammetry until two voltammograms were coincident. The probe oligo(1) was immobilized on to this activated electrode surface by adsorptive accumulation for $180 \mathrm{~s}$ applying a potential of $+0.50 \mathrm{~V}$ vs. $\mathrm{Ag} / \mathrm{AgCl}$, in a solution containing $0.28 \mu \mathrm{M}$ oligo(1). Then the probe oligo(1)-modified electrode was rinsed with acetate buffer.

\subsubsection{Hybridisation}

The hybridisation was performed by immersing the probe oligo(1)-modified electrode into a $0.8 \mu \mathrm{M}$ target oligo(2) solution for $300 \mathrm{~s}$ while holding the potential at $+0.5 \mathrm{~V}$. Then the electrode was rinsed with acetate buffer.

\subsubsection{Transduction}

Transduction was always carried out in $\mathrm{pH} 4.50 .1 \mathrm{M}$ acetate buffer using differential pulse voltammetry or electrochemical impedance spectroscopy at $+0.5 \mathrm{~V}$.

\subsubsection{Control}

Two control procedures were used before transduction:

1. Probe oligo(1)-modified electrode was immersed for 300 $\mathrm{s}$ into a solution containing $0.8 \mu \mathrm{M}$ non-complementary oligo(3) while the potential was held at $+0.50 \mathrm{~V}$.

2. The duplex oligo(1)-oligo(2) was adsorbed on the electrode surface for $180 \mathrm{~s}$ from a $0.26 \mu \mathrm{M}$ duplex solution while the potential was held at $+0.5 \mathrm{~V}$.

During all these steps except transduction the solutions were under continuous stirring.

\section{Results and discussion}

\subsection{AFM characterisation of the adsorbed oligonucleotides}

The process of adsorption of different oligonucleotide sequences was studied by MAC Mode AFM in air, in order to morphologically characterize the oligonucleotide-modified electrode surface.

In all AFM experiments, the HOPG substrate was used as working electrode. The extremely smooth, inert in air and easy to clean terraces of the HOPG electrode basal plane are an important advantage in studying adsorbed biological molecules. While the glassy carbon presents a root-meansquare (r.m.s.) roughness of $2.10 \mathrm{~nm}$, the HOPG electrode surface has an r.m.s. roughness of less than $0.06 \mathrm{~nm}$, for a $1000 \times 1000 \mathrm{~nm}^{2}$ surface area $[22,24]$.

The adsorption of the oligonucleotide sequences was performed during $180 \mathrm{~s}$, by applying an adsorption potential of $+0.65 \mathrm{~V}$, vs. AgQRE, as described in the Experimental section.

The first approach was to analyse the HOPG electrode modified by a thin film of an oligonucleotide sequence obtained by adsorption of $0.284 \mu \mathrm{M}(0.88 \mu \mathrm{g} / \mathrm{ml})$ oligo(1), the probe sequence. The surface as observed in the MAC Mode AFM images, Fig. 1A-1), confirmed the high capacity of single-stranded oligo(1) molecules to interact with the HOPG surface. The electrode appeared covered by a wellspread, uniform, but incomplete film, with a high degree of compactness, as observed in the section analysis from Fig. 1A-2). However, the oligo(1)-modified electrode surface layer presented holes, which correspond to the darker regions in the three dimensional image, that left the HOPG electrode surface exposed underneath.

Adsorption from a solution of $0.284 \mu \mathrm{M}(0.84 \mu \mathrm{g} / \mathrm{ml})$ oligo(2), the target, complementary sequence of oligo(1), also led to an incomplete network film onto HOPG surface, as observed in Fig. 1B. The network showed coiled fibres which joined together in end-to-end aggregations. The oligonucleotides condensed leaving large parts of the HOPG surface free.

Carrying out the adsorption from a solution $0.284 \mu \mathrm{M}$ $(0.83 \mu \mathrm{g} / \mathrm{ml})$ oligo(3), the non-complementary sequence of oligo(1), a small degree of surface coverage was again observed. The adsorbed oligo(3) molecules were stacked on the HOPG surface and appeared in the MAC Mode AFM images as small rods, Fig. 1C. The difference in the adsorption pattern of oligo(2) and oligo(3) pyrimidine-type oligonucleotides is probably due to differences in the oligonucleotide structure.

The first and most important step in DNA hybridisation detection experiments consists of the adsorption of the oligonucleotide probe sequence at the carbon electrode surface. Immobilisation of DNA probe on the sensing surface, while keeping the maximum conformational flexibility, is fundamental for the successful preparation of DNA biosensors. In order to obtain a DNA electrochemical 

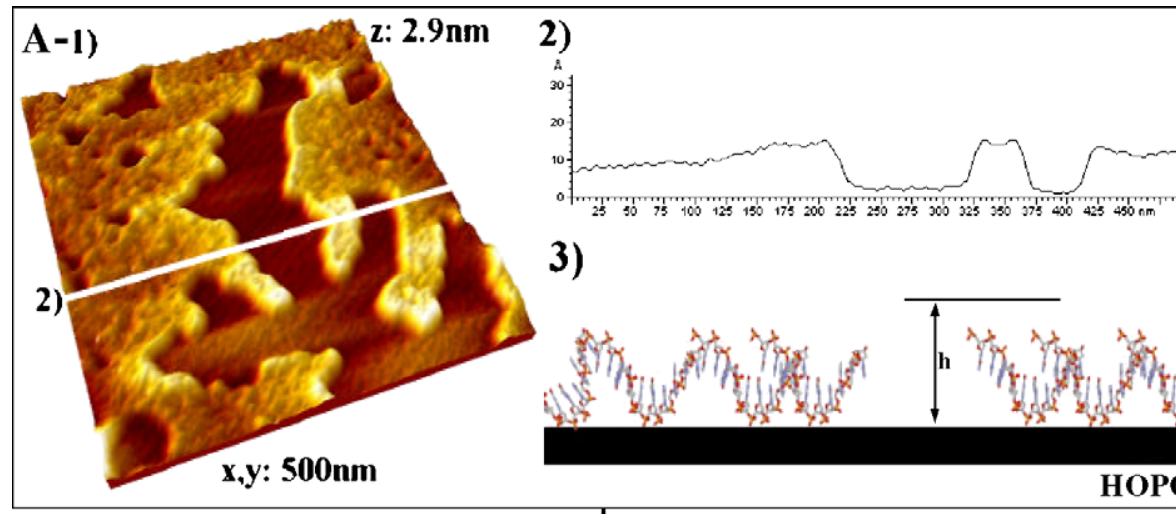

\section{3)}
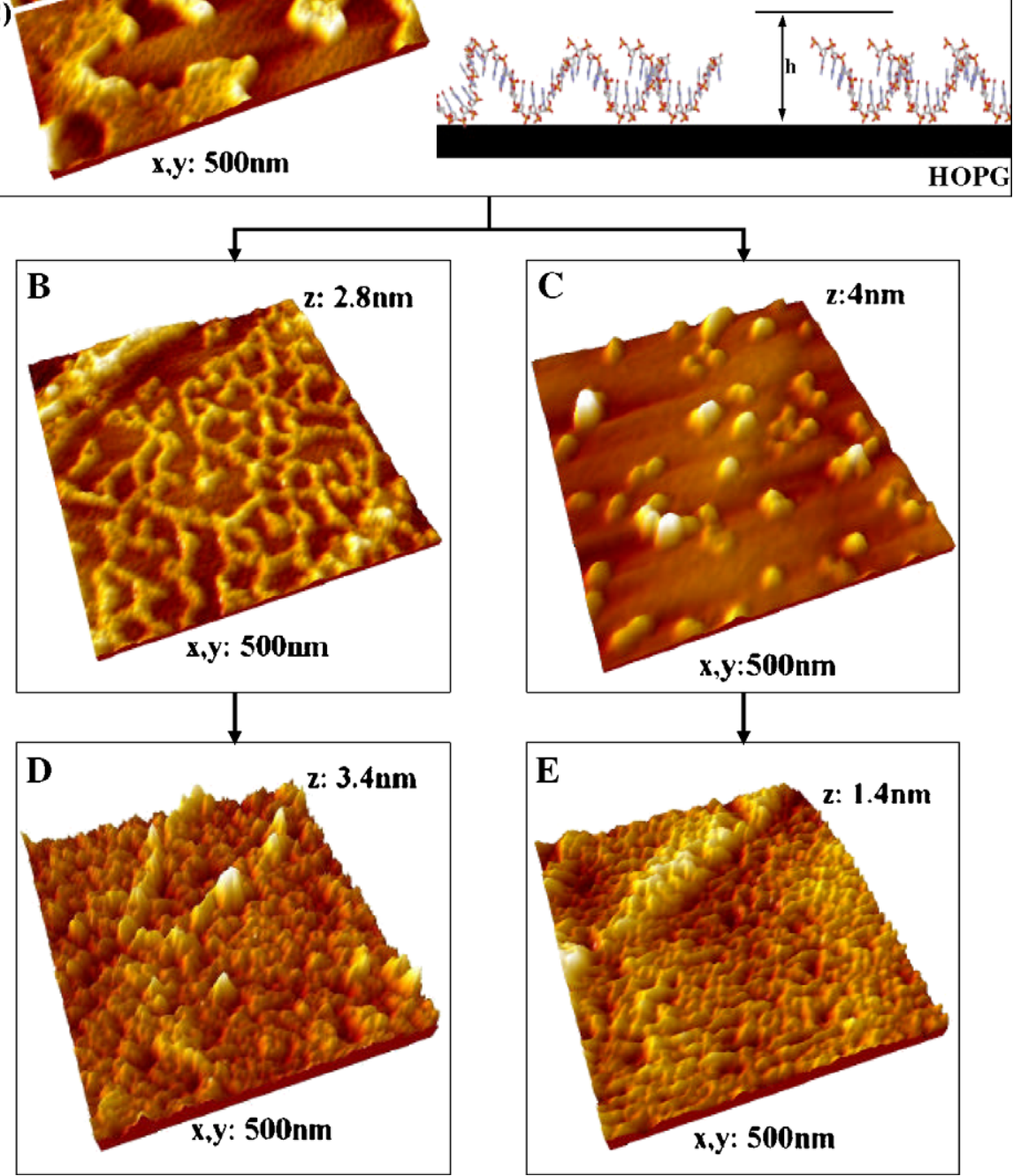

Fig. 1. MAC Mode AFM 3D images in air showing the adsorption, after applying a adsorption potential of $+0.65 \mathrm{~V}$, vs. AgQRE, of DNA oligonucleotides in $\mathrm{pH} 4.50 .1 \mathrm{M}$ acetate buffer electrolyte onto the HOPG electrode surface. (A-C) Adsorption on the HOPG electrode immersed into $0.284 \mu \mathrm{M}$ of (A-1) oligo(1), (B) oligo(2), and (C) oligo(3) sequences, after applying the adsorption potential for $180 \mathrm{~s}$. (A-2) Cross-section profile through the white line in the image (A-1). (A-3) Schematic diagram showing the adsorption of oligo(1) on the HOPG electrode surface. (D, E) Final adsorption on the HOPG electrode, first immersed in a $0.284 \mu \mathrm{M}$ oligo(1) and applying the adsorption potential for $180 \mathrm{~s}$, followed by immersion and applying the adsorption potential for $300 \mathrm{~s}$ in a $0.8 \mu \mathrm{M}$ : (D) oligo(2) and (E) oligo(3)

biosensor with high stability, selectivity and sensitivity necessary for the hybridisation and electrochemical detection of the process, the orientation and the packing of the DNA probe adsorbed layer is essential. Ideally, the oligonucleotide probes must be connected with the electrode surface at one point only, oriented with the sequences perpendicularly to the electrode surface in such a way that the complementary target sequence will have complete access to the immobilised probe, and sufficiently separated one from another to be able to enable hybridisation.
The potential of $+0.65 \mathrm{mV}$ vs. AgQRE, applied to the HOPG electrode, enhanced the stability of the DNA molecules at the electrode surface, due to the electrostatic interaction between the positive electrode and the negative sugar-phosphate DNA backbone. The oligonucleotides were strongly adsorbed under conditions of applied potential, leading to uniform, stable and reproducible MAC Mode AFM images, Fig. 1A-1) and each molecule could be attached at the surface by several sites, Fig. 1A-3). 
The DNA hybridisation efficiency is directly dependent on the accessibility of the DNA target to the DNA probe immobilized on the electrode surface, and consequently is dependent on the orientation of the DNA probe, perpendicular or parallel, on the electrode surface. The strong and compact DNA probe film observed, Fig. 1A-1), prevents hybridisation of many of the oligonucleotide sequences and, consequently, reduces the hybridisation efficiency.

Another critical issue in the development of a DNA electrochemical biosensor is to avoid non-specific adsorption of the target DNA sequences, since the electrochemical response of the DNA biosensor can be influenced by the electrochemical response of the target sequence. The high specificity and selectivity required by the DNA electrochemical biosensor is achieved by control and minimization of the non-specific adsorption on the electrodes.

Ideally, the oligonucleotide probe must be sufficiently densely packed to prevent the non-specific adsorption of the target between the probe molecules. However, as observed in the MAC Mode AFM images, due to the extensive existence of pores on the oligo(1)-modified electrode, large areas of the HOPG surface are not covered by the oligonucleotide probe molecular film, Fig. 1A.

The fact that the electrode is not completely covered allows the diffusion of other oligonucleotide molecules from the bulk solution to the surface and leads to nonspecific adsorption. Both complementary and non-complementary oligonucleotides adsorb at the HOPG surface, Fig. $1 \mathrm{~B}$ and $\mathrm{C}$, and adsorb non-specifically on the uncovered areas of the electrode left after the oligonucleotide probe immobilisation.

The evaluation of the surface coverage after hybridisation is also very important. For this reason, the hybridisation procedure with the complementary oligonucleotide, in the AFM electrochemical cell, was performed. The surface coverage was analysed by MAC Mode AFM in air.

First, the HOPG electrode was modified by adsorption of the probe oligo(1), applying the adsorption potential for $180 \mathrm{~s}$, in a $0.284 \mu \mathrm{M}(0.88 \mu \mathrm{g} / \mathrm{ml})$ oligo(1) solution and an oligo(1)-modified HOPG electrode was obtained, Fig. 1A1). This procedure was followed by the hybridisation step, which consisted in applying the adsorption potential for $300 \mathrm{~s}$, to the HOPG electrode immersed into $0.8 \mu \mathrm{M}(2.35$ $\mu \mathrm{g} / \mathrm{ml}$ ) oligo(2) target complementary sequence solution, Fig. 1D.

After performing the hybridisation on the oligo(1)modified HOPG electrode surface, the MAC Mode AFM image from Fig. 1D shows that the molecules self-organize in a dense, thick and uniform film of DNA, covering the electrode completely, and not leaving any pores.

Ideally, after the DNA hybridisation is completed, all the probe molecules should be hybridised. However, the surface topography observed was formed by adsorbed hybrid double-stranded oligo(1)-oligo(2) molecules, together with adsorbed single-stranded oligo(1) and single-stranded oligo(2) molecules, forming a compact film.
Consequently, from the present MAC Mode AFM data it is very difficult to distinguish the binding specificity in the DNA hybridisation.

In a control experiment the same hybridisation procedure was performed, but instead of the target complementary oligo(2) sequence the non-complementary oligo(3) sequence was used. After adsorption of probe oligo(1) molecules for $180 \mathrm{~s}$, the oligo(1)-modified HOPG electrode was immersed into a $0.8 \mathrm{mM}(2.35 \mu \mathrm{g} / \mathrm{ml})$ oligo(3) noncomplementary solution, and the adsorption potential was applied during $300 \mathrm{~s}$. After analysing the surface by MAC Mode AFM, it was also observed that the HOPG electrode had a complete coverage with DNA molecules, Fig. 1E.

After both hybridisation, Fig. 1D, and control, Fig. 1E, experiments the observed MAC Mode AFM images showed insignificant differences in surface coverage. However the density of molecules at the surface is much higher than the number of molecules that are hybridised at the surface, since the concentration of oligo(2) and oligo(3) is more than double of oligo(1). Consequently MAC Mode AFM study showed that the level of non-specific adsorption at the HOPG electrode surface is quite high, giving rise to a featureless image and making it impossible to distinguish between the hybridised and non-hybridised molecules on the HOPG surface, Fig. 1D, E.

\subsection{Voltammetric studies in incubated solutions}

The first hybridisation experiments were carried out in incubated solutions containing the complementary oligonucleotides (1) and (2) and differential pulse voltammograms were recorded in $\mathrm{pH} 4.50 .1 \mathrm{M}$ acetate buffer containing 0.8 $\mu \mathrm{M}$ probe oligo(1) and $0.8 \mu \mathrm{M}$ target complementary oligo(2), before and after different hybridisation periods, Fig. 2A. The electrode surface was polished between each measurement to avoid current drops due to the strong adsorption of oligonucleotides on the glassy carbon surface. A gradual decrease of both guanosine and adenosine oxidation peaks with incubation time was observed and constant peak currents were obtained only after $24 \mathrm{~h}$ of hybridisation in solution.

At the same time, control experiments consisting of 0.8 $\mu \mathrm{M}$ probe oligo(1) incubated for different time intervals with $0.8 \mu \mathrm{M}$ non-complementary oligo(3) were also carried out. Differential pulse voltammograms, Fig. 2B, always using freshly polished electrodes, showed very small changes of the oxidation peak currents of guanosine and adenosine residues even for very long time intervals. But, since small differences were also observed in the base line recorded after polishing the electrode they could be responsible for the small fluctuations of the current peaks presented in Fig. 2B.

This experiment showed that in solution probe oligo(1) hybridised with its complementary target oligo(2) and not with the non-complementary oligo(3) and the process could be followed by differential pulse voltammetry. 
A

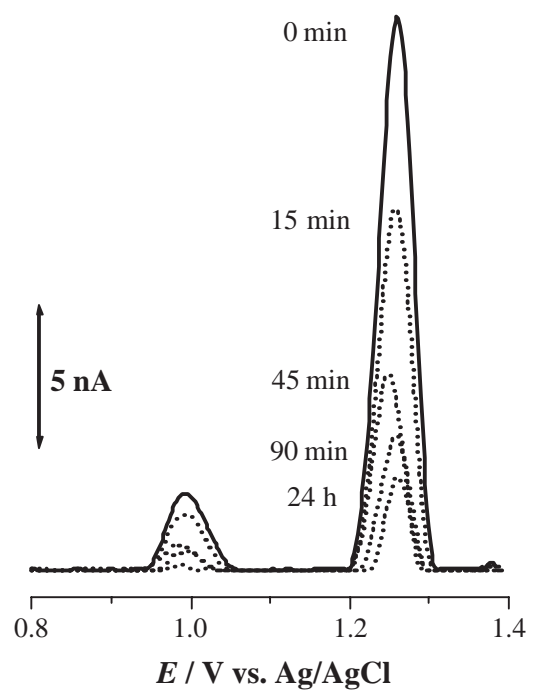

B

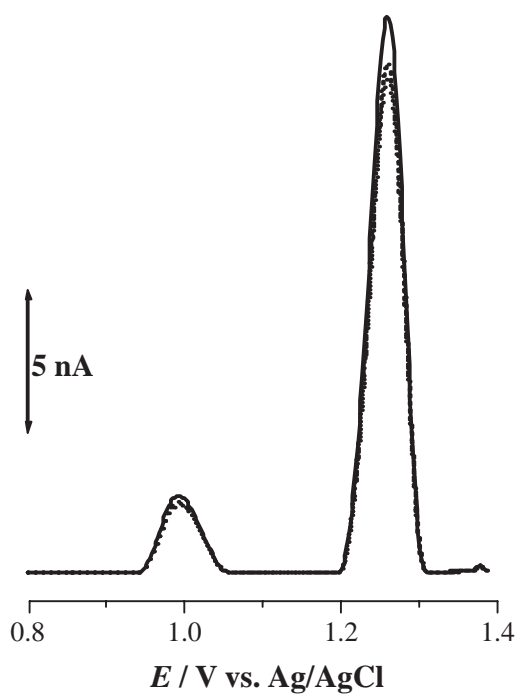

Fig. 2. DP voltammograms obtained in a mixture of $0.8 \mu \mathrm{M}$ oligo(1) with: (A) $0.8 \mu \mathrm{M}$ complementary oligo(2) after different hybridisation times. (B) $0.8 \mu \mathrm{M}$ non-complementary oligo(3) after: 0,1 and $24 \mathrm{~h}$. Supporting electrolyte $\mathrm{pH} 4.50 .1 \mathrm{M}$ acetate buffer. Scan rate $5 \mathrm{mV} \mathrm{s}{ }^{-1}$, pulse amplitude $50 \mathrm{mV}$, pulse width $70 \mathrm{~ms}$.

The limitations of such an experiment are obvious and immobilisation of a known oligonucleotide sequence onto an electrode surface followed by hybridisation in the complementary solution and transduction in pure electrolyte was investigated. However, much attention has to be paid to the probe immobilisation conditions in order to reduce the contribution of non-specifically adsorbed oligonucleotides [7].

\subsection{Voltammetric studies of the adsorption of probe oligo(1)}

Prior to hybridisation experiments, the adsorption properties of probe oligo(1) at a GCE surface were investigated.

Different probe oligo(1) concentrations, between 0.02 and $0.5 \mu \mathrm{M}$, were assessed in connection with different adsorption times, 10-400 s, using an applied adsorption potential of $+0.50 \mathrm{~V}$. Guanosine and adenosine peak currents obtained in buffer after adsorption of oligo(1) at the adsorption potential for $180 \mathrm{~s}$ in solutions of different concentration of oligo(1) are shown in Fig. 3A. A linear increase of the guanosine and adenosine peak currents was found to take place when the oligo(1) concentration was varied between 0.05 and $0.5 \mu \mathrm{M}$. After fitting the curves, this enabled the calculation of the detection limits of adsorbed oligo(1) based guanosine and adenosine peak currents. It was found to be $6.6 \times 10^{-9} \mathrm{M}$ for guanosine and $3.5 \times 10^{-8} \mathrm{M}$ for adenosine.

The effect of adsorption time on the oligo(1) guanosine and adenosine current peaks is presented in Fig. 3B. Adsorption for different times at an applied potential of
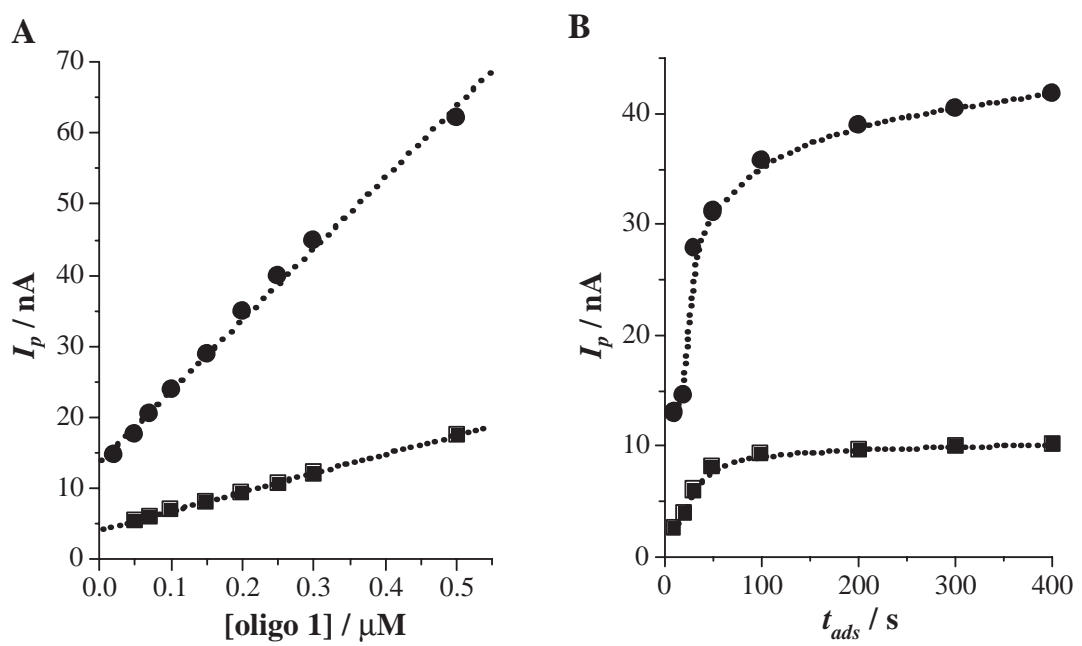

Fig. 3. Oxidation peak currents in pH 4.5 0.1 M acetate buffer for ( $\mathbf{a})$ guanosine and ( $)$ adenosine obtained after adsorption of oligo(1) onto the electrode surface during: (A) $3 \mathrm{~min}$ in different concentrated solutions. (B) Different adsorption times from a solution of $0.28 \mu \mathrm{M}$. 
$+0.50 \mathrm{~V}$ from a solution containing $0.28 \mu \mathrm{M}$ oligo(1), followed by the detection in buffer, showed a progressive increase of the guanosine and adenosine current peaks during the first $60 \mathrm{~s}$ of adsorption, but afterwards the currents remained constant at $10 \mathrm{nA}$ for guanosine and 40 $\mathrm{nA}$ for adenosine.

From these results, it was considered that $180 \mathrm{~s}$ adsorption, at an applied potential of $+0.5 \mathrm{~V}$, in a 0.28 $\mu \mathrm{M}$ solution of probe oligo(1) ensured a good coverage of the electrode surface and therefore these conditions have been used for further evaluation of the hybridisation experiments carried out at the GCE surface.

\subsection{Voltammetric characterization of hybridisation on the modified electrode surface}

Differential pulse voltammograms of probe oligo(1)modified GCE before and after immersion of the electrode into solutions containing target oligo(2) or non-complementary oligo(3) were recorded in buffer, Fig. 4A. After each medium exchange the electrode was thoroughly washed with deionized water to assure the removal of unbound molecules, and between each series of experiments the electrode surface was always polished.

Immobilisation of the probe oligo(1) at the GCE surface was followed by transduction in buffer and showed the oxidation peaks corresponding to the guanosine and adenosine present in the oligonucleotide sequence, Fig. 4A (dotted line).

In another experiment, immobilisation of the probe oligo(1) at the GCE surface was followed by immersing the probe oligo(1)-modified GCE surface into $0.8 \mu \mathrm{M}$ target oligo(2) solution, during $300 \mathrm{~s}$ while the potential was kept constant at $+0.5 \mathrm{~V}$, and allowing hybridisation to occur. After this, the electrode was washed and transferred to buffer solution and a big decrease of both guanosine and adenosine oxidation peaks was observed, Fig. 4A (dash-dot line). As the hybridisation reaction occurred hydrogen bonds between complementary bases were formed giving rise to the duplex form, more difficult to oxidize.

In a control experiment, a differential pulse voltammogram was recorded in buffer, after adsorption from a 0.26 $\mu \mathrm{M}$ duplex oligo(1)-oligo(2) solution, for $180 \mathrm{~s}$ at an applied potential of $+0.5 \mathrm{~V}$, Fig. 4A (full line). The guanosine and adenosine oxidation peak currents of the adsorbed duplex oligo(1)-oligo(2) can be easily compared with those obtained after the previous hybridisation experiment. In both cases smaller peaks for guanosine and adenosine were recorded as expected.

The variation of guanosine and adenosine oxidation peak currents with hybridisation time was also monitored, Fig. 4B. Both guanosine and adenosine peaks decreased rapidly during the first $300 \mathrm{~s}$ of hybridisation. The adenosine oxidation peak reached a constant value of about $10 \mathrm{nA}$ after $300 \mathrm{~s}$ of hybridisation whereas the guanosine peak almost disappeared, for longer hybridisation periods. An explanation for this effect can be the composition of the probe oligo(1) sequence $5^{\prime}$-AAAGAAAAAG-3' with 8 adenines and only 2 guanines.

Control experiments were also carried out using first the non-complementary oligo(3) instead of the target complementary oligo(2).

The probe oligo(1)-modified electrode was held at +0.5 $\mathrm{V}$ for $300 \mathrm{~s}$ in a solution containing $0.8 \mu \mathrm{M}$ noncomplementary oligo(3). The electrode was then transferred to buffer and the differential pulse voltammogram recorded,
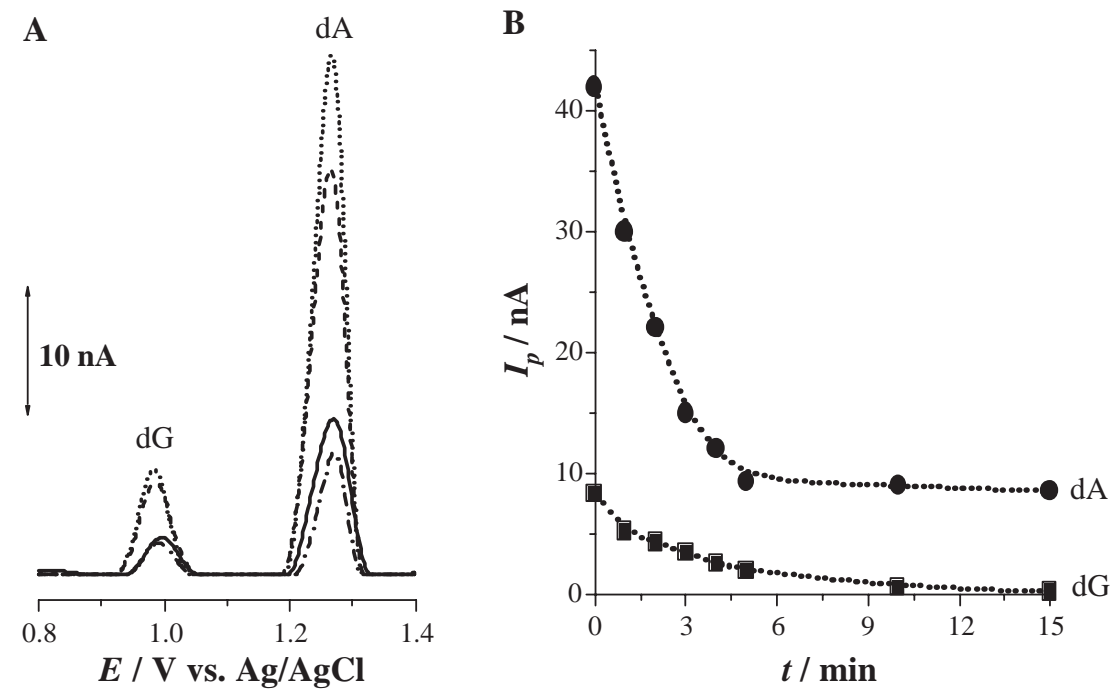

Fig. 4. (A) DP voltammograms obtained in $\mathrm{pH} 4.50 .1 \mathrm{M}$ acetate buffer after: (-) adsorption at $+0.5 \mathrm{~V}$ for 3 min in a solution containing $0.26 \mu \mathrm{M}$ duplex,

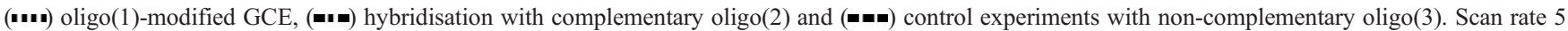
$\mathrm{mV} \mathrm{s}^{-1}$, pulse amplitude $50 \mathrm{mV}$, pulse width $70 \mathrm{~ms}$. (B) Variation of (⿴) guanosine and ( ) adenosine oxidation current peaks with hybridisation time of complementary oligo(2) with oligo(1)-modified GCE. 
Fig. 4A (dashed line). Both guanosine and adenosine oxidation current peaks decreased (more in the case of adenosine) when compared with the differential pulse voltammogram obtained after immobilisation of probe oligo(1), Fig. 4A (dotted line). Since no hybridisation could have occurred with the non-complementary oligo(3) during the control experiment, the decrease of the peaks needs to be explained.

DNA strongly adsorbs at the electrode surface in a network pattern such that regions of the bare electrode surface are exposed to the solution [22]. Therefore, during the control experiments some of the non-complementary oligo(3) adsorb on uncovered regions of probe oligo(1)modified electrode, Fig. 1A, and some displacement of probe oligo(1) from the electrode surface by oligo(3) could have occurred. This experiment was repeated several times and good reproducibility was obtained. Electrode surface renewal by polishing between successive scans only caused small fluctuations in the background when using a freshly polished electrode surface and cannot be responsible for this effect which does not affect the qualitative results.

The differential pulse voltammetric experiments showed that after the hybridisation reaction a decrease in the guanosine and adenosine oxidation peak currents occurred as expected. However, an unexpected current drop was observed for longer hybridisation periods and during control experiments which could be due to non-specific adsorption.

The effect of non-specific adsorption could not be evaluated using only voltammetric experiments due to the nature of the target and non-complementary oligonucleotides and therefore further investigation of the properties of the interfacial region formed at the electrode surface was carried out.

\subsection{Impedance characterization of hybridisation on the modified electrode surface}

Electrochemical impedance spectroscopy measurements were performed in order to characterize the electrical double layer formed after immobilisation, hybridisation and control experiments, of the oligonucleotide sequences.

The frequency dependence of the impedance spectra of probe oligo(1)-modified electrode in a complex impedance plot before and after hybridisation, and after control experiments are shown in Fig. 5A. All measurements were performed at an applied potential of $+0.5 \mathrm{~V}$. The sequentially recorded spectra after immobilisation, hybridisation or control experiments, showed no different features which confirms that during these measurements no detectable desorption of the oligonucleotide sequences from the electrode surface takes place.

The complex impedance is composed of a real $\left(Z^{\prime}\right)$ and an imaginary part $\left(Z^{\prime \prime}\right)$ that provides direct information about the overall capacitance of the electrode double layer [25]. A pure capacitive behaviour of the probe oligo(1)modified electrode surface was observed after adsorption for $180 \mathrm{~s}$ at an applied potential of $+0.5 \mathrm{~V}$ from a solution containing $0.28 \mu \mathrm{M}$ oligo(1).

Following the hybridisation procedure described in the Experimental section an increase in the impedance occurred, corresponding to a decrease of the capacitance of the electrode double-layer, since in these cases the impedance follows a relationship of inverse proportionality with the capacitance of the system.

Control experiments were carried out. The impedance spectrum obtained for the control is observed as being intermediate between that recorded at the probe oligo(1)modified electrode and the impedance spectrum obtained after hybridisation.
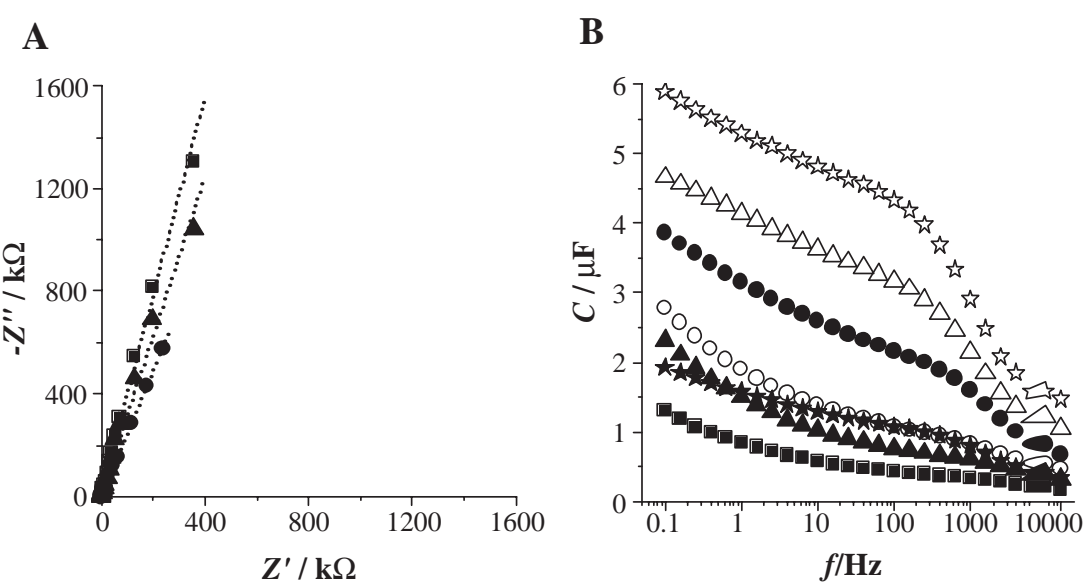

Fig. 5. (A) Impedance spectra in pH 4.5 0.1 M acetate buffer at fixed potential $E=+0.5 \mathrm{~V}$ using a $5 \mathrm{mV}$ r.m.s. amplitude obtained from: (O) oligo(1)-modified GCE, ( $\mathbf{\square})$ after hybridisation with oligo(2) and ( $\boldsymbol{\Delta}$ ) control experiments with oligo(3). (B) Capacitance data from impedance spectra obtained for: (放) bare GCE, ( $\star$ ) GCE modified by duplex adsorption; and after adsorption of $0.28 \mu \mathrm{M}$ oligo(1) during $(\bigcirc) 3$ and $(\bigcirc) 5$ min, $(\triangle)$ after adsorption of $0.8 \mu \mathrm{M}$ oligo(2) during 5 min, ( $\mathbf{\square})$ after hybridisation of complementary oligo(2) with oligo(1)-modified GCE and ( $\mathbf{\Delta})$ after control experiment of non-complementary oligo(3) with oligo(1)-modified GCE. 
The capacitive behaviour of the system can be more conveniently represented using the capacitance data obtained from the impedance spectra. Changes taking place in the double layer capacitance during immobilisation, hybridisation and control experiments were plotted in function of the applied a.c. perturbation frequency, Fig. 5B.

The capacitance variation with frequency of the GCE double layer was recorded before and after adsorption at an applied potential of $+0.5 \mathrm{~V}$ for different time intervals in a solution containing $0.28 \mu \mathrm{M}$ probe oligo(1). The changes in the capacitance could be easily followed for low frequencies. In these conditions, modifications in the double layer were taking place slowly and the initial conformation (before the perturbation was applied) was reached before the system is disturbed again.

The capacitance data obtained from the impedance spectra after adsorption for 180 and $300 \mathrm{~s}$ in a solution containing $0.28 \mu \mathrm{M}$ probe oligo(1) is shown in Fig. 5B. The immobilisation of probe oligo(1) at the electrode surface leads to a decrease in the capacitance when compared with that of the surface of a bare GCE. Lower values of the capacitance were achieved if the adsorption time was increased.

The data obtained from the impedance spectra after adsorption for $300 \mathrm{~s}$ in a solution containing $0.8 \mu \mathrm{M}$ target oligo(2), Fig. 5B, also show a decrease in the double layer capacitance. Although the changes obtained after adsorption in $0.8 \mu \mathrm{M}$ target oligo(2) are not so pronounced as in the case of adsorption from a less concentrated $0.3 \mu \mathrm{M}$ probe oligo(1) solution the capacitance data clearly demonstrated that oligonucleotides containing only pyrimidinic bases also adsorb at the GCE surface.

Following the hybridisation procedure described in the Experimental section a large drop in the capacitance values was again observed. As the hybridisation reaction proceeds changes take place both in the thickness of the double layer and the medium permittivity, in such a way that the capacity value is decreasing [26].

However, after adsorption at $+0.5 \mathrm{~V}$ for $180 \mathrm{~s}$ from a $0.26 \mu \mathrm{M}$ duplex solution, a higher value for the capacitance was obtained. Comparing the capacitance data obtained from the impedance spectrum of the duplex with the spectrum obtained after hybridisation it can be concluded that during the hybridisation, besides the double strand formation, non-specific adsorption of the complementary sequence in the uncovered regions took place forming a mixed multilayer. The difference of capacitances measured at high and low frequency in the double helix is not so clear, due to its greater rigidity, as observed in the single stranded oligonucleotides.

The contribution from non-specific adsorption to the overall capacitance change was verified in the control experiments performed using oligo(3). In the control experiment the capacitance obtained from the impedance spectra after adsorption for $180 \mathrm{~s}$ of probe oligo(1), Fig. 5B ( $\star$ ) was first determined. After this, oligo(1)-modified electrode was immersed in for $180 \mathrm{~s}$ in a solution of oligo(3). A decrease of the capacitance after adsorption for $180 \mathrm{~s}$ of oligo(3) onto the oligo(1)-modified electrode was observed when compared with that obtained after adsorption for $180 \mathrm{~s}$ of probe oligo(1). Since no hybridisation could have occurred during this experiment the capacitance decrease can only be attributed to non-specific adsorption of the non-complementary oligo(3).

\section{Conclusions}

The results clearly show the importance of characterising the surface morphology and of understanding the adsorption process of oligonucleotides on the carbon electrode surface, in the evaluation of all the factors that can influence the correct response of DNA electrochemical biosensors. The MAC Mode AFM images of oligonucleotide films formed under controlled potential showed the existence of extensive uncovered areas on the electrode surface. This aspect must be carefully considered when using unmodified DNA electrochemical biosensors for the detection of DNA hybridisation processes, as it enables the non-specific adsorption of molecules on the uncovered areas.

Hybridisation experiments carried out in solution showed that the recognition process between two complementary oligonucleotides occurs slowly and it could be followed by differential pulse voltammetry. Hybridisation and control experiments carried out on the electrode surface showed a decrease in the oxidation currents of guanosine and adenosine residues, but the changes in the oxidation current are due to hybridisation events as well as to non-specific adsorption that cause also blockage of the electrode surface.

During the hybridisation procedure, a mixed DNA multilayer was formed on the electrode surface, due to double strand formation and to non-specific adsorption of complementary DNA sequence. Avoiding and protecting the biosensor from non-specific adsorption continue to be a very important goal to achieve when developing reliable electrochemical hybridisation biosensors.

\section{Acknowledgments}

Financial support from Fundação para a Ciência Tecnologia (FCT), Ph.D. Grant PRAXIS SFRH/BD/877/2000 (V.C.D.) and Post-Doc Grant SFRH/BPD/14425/2003 (A.M. C.P.), POCTI (co-financed by the European Community Fund FEDER), ICEMS (Research Unit 103), and European Projects QLK3-2000-01311 and HPRN-CT-2002-00186 are gratefully acknowledged.

\section{References}

[1] K.M. Millan, S.R. Mikkelsen, Sequence-selective biosensor for DNA based on electroactive hybridisation indicators, Anal. Chem. 65 (1993) 2317-2322. 
[2] J. Wang, X. Cai, G. Rivas, H. Shiraishi, P.A.M. Farias, N. Dontha, DNA electrochemical biosensor for the detection of short DNA sequences related to the human immunodeficiency virus, Anal. Chem. 68 (1996) 2629-2634.

[3] A. Erdem, K. Kerman, B. Meric, U.S. Akarca, M. Ozsoz, Novel hybridisation indicator methylene blue for the electrochemical detection of short DNA sequences related to the hepatitis B virus, Anal. Chim. Acta 422 (2000) 139-149.

[4] J. Wang, A.N. Kawde, Pencil-based renewable biosensor for label-free electrochemical detection of DNA hybridisation, Anal. Chim. Acta 431 (2001) 219-224.

[5] E. Palecek, M. Fojta, F. Jelen, New approaches in the development of DNA sensor: hybridisation and electrochemical detection of DNA and RNA at two different surfaces, Bioelectrochemistry 56 (2002) 85-90.

[6] J. Wang, G. Liu, A. Merkoci, Particle-based detection of DNA hybridisation using electrochemical stripping measurements of an iron tracer, Anal. Chim. Acta 482 (2003) 149-155.

[7] J. Wang, G. Rivas, J.R. Fernandes, J.L.L. Paz, M. Jiang, R. Waymire, Indicator-free electrochemical DNA hybridisation biosensor, Anal. Chim. Acta 375 (1998) 197-203.

[8] G. Marrazza, I. Chianella, M. Mascini, Disposable DNA electrochemical sensor for hybridisation detection, Biosen. Bioelectron. 14 (1999) 43-51.

[9] K. Kerman, Y. Morita, Y. Takamura, E. Tamiya, Label-free electrochemical detection of DNA hybridisation on gold electrode, Electrochem. Commun. 5 (2003) 887-891.

[10] M. Gheorghe, A.G. Elie, Electrochemical frequency dependent characterization of DNA hybridisation, Biosens. Bioelectron. 19 (2003) $95-102$.

[11] L. Strasak, J. Dvorak, S. Hason, V. Vetterl, Electrochemical impedance spectroscopy of polynucleotides adsorption, Bioelectrochemistry 56 (2002) $37-41$.

[12] V. Vetterl, N. Papadopoulos, V. Drazan, L. Strasak, S. Hason, J. Dvorak, Nucleic acid sensing by impedance measurements, Electrochim. Acta 45 (2000) 2961-2971.

[13] H. Su, M. Thompson, Kinetics of interfacial nucleic acid hybridisation studied by acoustic network analysis, Biosens. Bioelectron. 10 (1995) $329-340$

[14] C. Nicolini, V. Erokhin, P. Facci, S. Guerzoni, A. Ross, P. Paschkevitsch, Quartz balance DNA sensor, Biosens. Bioelectron. 12 (1997) 613-618.
[15] K. Ito, K. Hashimoto, Y. Ishimori, Quantitative analysis for solidphase hybridisation reaction and binding reaction of DNA binder to hybrids using a quartz crystal microbalance, Anal. Chim. Acta 327 (1996) 29-35.

[16] T. Ratilainen, A. Holmen, E. Tuite, G. Haaima, L. Christensen, P.E. Nielsen, B. Norden, Hybridisation of peptide nucleic acid, Biochem. 37 (1998) $12331-12342$.

[17] K.J. Breslauer, R. Frank, H. Blöcker, L.A. Marky, Predicting DNA duplex stability from the base sequence, Proc. Natl. Acad. Sci. U.S.A. 83 (1986) 3746-3750.

[18] P.A.E. Piunno, U.J. Krull, R.H.E. Hudson, M.J. Damha, H. Cohen, Fiber-optic DNA sensor for fluorometric nucleic acid determination, Anal. Chem. 67 (1995) 2635-2643.

[19] F. Kleinjung, F.F. Bier, A. Warsinke, F.W. Scheller, Fibre-optic genosensor for specific determination of femtomolar DNA oligomers, Anal. Chim. Acta 350 (1997) 51-58.

[20] J.H. Waterson, P.A.E. Piunno, U.J. Krull, Toward the optimization of an optical DNA sensor: control of selectivity coefficients and relative surface affinities, Anal. Chim. Acta 457 (2002) 29-38.

[21] J.H. Waterson, P.A.E. Piunno, U.J. Krull, Practical physical aspects of interfacial nucleic acid oligomer hybridisation for biosensor design, Anal. Chim. Acta 469 (2002) 115-127.

[22] A.M. Oliveira Brett, A.-M. Chiorcea, Atomic force microscopy of DNA immobilized onto a highly oriented pyrolytic graphite electrode surface, Langmuir 19 (2003) 3830-3839.

[23] A.M. Oliveira Brett, A.-M. Chiorcea, Effect of $\mathrm{pH}$ and applied potential on the adsorption of DNA on highly oriented pyrolytic graphite electrodes. Atomic force microscopy surface characterisation, Electrochem. Commun. 5 (2003) 178-183.

[24] A.M. Oliveira Brett, A.-M. Chiorcea, Atomic force microscopy characterization of an electrochemical DNA-biosensor, Bioelectrochemistry 63 (2004) 229-232.

[25] J.P. Cloarec, N. Deligianis, J.R. Martin, I. Lawrence, E. Souteyrand, C. Polychronakos, M.F. Lawrence, Immobilisation of homooligonucleotide probe layers onto $\mathrm{Si} / \mathrm{SiO}_{2}$ substrates: characterization by electrochemical impedance measurements and radiolabelling, Biosens. Bioelectron. 17 (2002) 405-412.

[26] F. Wei, B. Sun, W. Liao, J. Ouyang, X.S. Zhao, Achieving differentiation of single-base mutation through hairpin oligonucleotides and electric potential control, Biosens. Bioelectron. 18 (2003) $1149-1155$. 